\title{
Intracellular Acetylcholine Receptors in Skeletal Muscles of the Adult Rat $^{1}$
}

\author{
ALAN PESTRONK \\ Department of Neurology, Johns Hopkins University School of Medicine, Baltimore, Maryland 21205
}

\begin{abstract}
We have examined the localization and numbers of acetylcholine receptors (AChRs) within adult mammalian skeletal muscle using ${ }^{125} \mid-\alpha$-bungarotoxin $\left({ }^{125} \mid-\alpha\right.$-BuTx). Intracellular AChRs were exposed to ${ }^{125} \mid-\alpha$-BuTx by permeabilizing the muscle membrane with the detergent saponin. Our results show that, in muscles of young adult rats, internal AChRs occur in a number and pattern of distribution similar to that for surface membrane AChRs. Internal AChRs are found only in perijunctional regions of normally innervated muscles, but they appear all along the length of muscle fibers after denervation. A minority of internal AChRs may be newly synthesized AChRs en route to insertion in the surface membrane; however, most internal AChRs appear to be antigentically different from and to behave independently of surface membrane AChRs. With increasing age, internal AChRs decline in number, while surface AChRs remain relatively constant. In older animals, internal AChRs may reappear in muscles that have undergone degeneration and regeneration. We conclude that a significant proportion of AChRs in skeletal muscle may be intracellular. Internal AChRs are a useful marker for young muscle cells. Their distribution may provide information about the intracellular pathways of AChR metabolism.
\end{abstract}

Acetylcholine receptors (AChRs) in mammalian skeletal muscle are found both on the surface membrane and within the cell. Numerous studies of surface AChRs have shown that, in normal adult muscle, AChRs are located primarily at neuromuscular junctions (Barnard et al., 1971; Fambrough and Hartzell, 1972; Albuquerque et al., 1974; Fertuck and Salpeter, 1974; Daniels and Vogel, 1975). After denervation new AChRs appear in the extrajunctional surface membrane (Miledi and Potter, 1960; Hartzell and Fambrough, 1972; Libelius, 1974; Chang et al., 1975; Pestronk et al., 1976). Studies of internal AChRs in developing or cultured muscle have identified them, in relatively small numbers, along the length of fibers associated with Golgi apparatus and multivesicular bodies (Fambrough and Devreotes, 1978; Atsumi, 1981). It has been proposed that internal AChRs represent either newly synthesized AChRs, in transport to the surface, or internalized AChRs en route to lysosomal degradation (Fumagalli et al., 1982). However, there have been few studies of internal AChRs in adult muscle; little is known about their

Received April 23, 1984; Revised October 15, 1984 Accepted November 8, 1984

\footnotetext{
${ }^{1}$ This work was supported by Grants 5P01 NS109020 and 5R01 HD04817 from the National Institutes of Health. I am grateful to D. B. Drachman for helpful discussions on the manuscript, to M. J. Peper and R. N. Adams for technical assistance, and to C. F. Barlow-Salemi and R. A. White for assistance in the preparation of the manuscript.
}

distribution or their changes with aging or denervation. Definition of the number and localization of internal AChRs should provide information about the intracellular pathways associated with the patterns of distribution of surface AChRs.

In this study, we have examined internal AChRs in vivo, in normal and denervated adult rat skeletal muscle, and in vitro, in cultured rat skeletal muscle. Internal AChRs were exposed by permeabilizing membranes in paraformaldehyde-fixed muscle using the detergent saponin and were labeled with ${ }^{125} \mid-\alpha$-bungarotoxin ( $\alpha$-BuTx). Our results show that, in the muscles of 3-to 7-month old rats, there are large numbers of internal $\alpha$-BuTx binding sites with properties similar to AChRs. The distribution of these sites parallels that of surface AChRs in innervated and denervated muscle. After 7 months of age, internal sites decline in number but they recur in regenerated muscle fibers.

\section{Materials and Methods}

Most experimental procedures were carried out using female SpragueDawley rats (aged 1 to 18 months). Female Lewis rats (aged 3 to 4 months) were used in the anti-AChR antibody experiments. AChRs from the electric organ of Torpedo californicus were purified by affinity chromatography (Pestronk et al., 1983). Anti-AChR antibodies were raised in Lewis rats by immunization with $50 \mu \mathrm{g}$ of AChR, emulsified in an equal volume of Freund's complete adjuvant, and injected intradermally. Anti-Torpedo AChR antibody titers averaged $1685 \pm 218 \times 10^{-9} \mathrm{M}$ at the time soleus muscles were removed for study. $\alpha$-BuTx (Miami Serpentarium) was repurified by ionexchange chromatography using a Whatman $\mathrm{CM}-32$ column (Lee et al. 1972).

Muscle fixation. Muscles were pinned at resting length in dishes containing modified Hams F-12 culture medium, teased longitudinally into thin strips, and incubated for $30 \mathrm{~min}$ in a $2 \%$ paraformaldehyde fixative solution that preserves $\alpha$-BuTx binding to AChRs (Fambrough and Devreotes, 1978). (The fixative was prepared as follows: paraformaldehyde was dissolved in $20 \mathrm{~mm}$ sodium phosphate buffer, $\mathrm{pH} 7.2\left(80\right.$ to $\left.85^{\circ} \mathrm{C}\right)$, to make a $2 \%$ formaldehyde solution. This was allowed to cool, and lysine (as the free base; finat concentration, $100 \mathrm{~mm}$ ) and sucrose (final concentration, $60 \mathrm{~mm}$ ) were added. The solution was reheated $\left(80^{\circ} \mathrm{C}\right)$, and sodium periodate was added (final concentration, $10 \mathrm{~mm}$ ).) After returning to room temperature, the fixative could be used immediately or kept refrigerated for several days. Before labeling with ${ }^{125} \mathrm{k}-\alpha$-BuTx, muscles were washed twice for $10 \mathrm{~min}$ in the culture medium.

Exposure of internal AChRs. To allow $\alpha$-BuTx access to intracellular AChRs muscle membranes were permeabilized using saponin. Fixed muscle was treated for 6 min with $0.5 \%$ saponin in $20 \mathrm{~mm}$ sodium phosphate buffer, $\mathrm{pH}$ 7.2. Muscles were then washed three times in culture medium over 15 min.

Labeling of AChRs. To label AChRs with ${ }^{125}$ - $\alpha$-BuTx fresh, fixed, or fixedsaponin treated muscles were: (1) incubated for 3 to $4 \mathrm{hr}$ at $37^{\circ} \mathrm{C}$ in culture medium containing 0.1 to $0.15 \mu \mathrm{g} / \mathrm{ml}^{125} \mid-\alpha$-BuTx (specific activity, 2 to $6 \times$ $10^{4} \mathrm{Ci} / \mathrm{mol}$ ); (2) rinsed 12 times at 5 -min intervals with Hanks' balanced salt solution containing $0.5 \%$ bovine serum albumin (fraction $\mathrm{V}$ ) buffered at $\mathrm{pH}$ 7.2 with HEPES, $4.3 \mathrm{gm} /$ liter; allowed to stand in a refrigerator at $4^{\circ} \mathrm{C}$ overnight; washed 5 more times; and (3) fixed in 2.5 to $5 \%$ glutaraldehyde for $30 \mathrm{~min}$ to $3 \mathrm{hr}$ to allow subsequent teasing of the muscle fiber bundles.

Quantitation of AChRs. Neuromuscular junctions (NMJs) were localized by teasing longitudinal strips of muscle containing 200 to 600 fibers and 
staining them for acetylcholinesterase (AChE) (Pestronk et al., 1976). Long muscle strips containing AChE-stained NMJs were then further dissected under the stereomicroscope into small bundles of 50 to 100 fibers, and the number of fibers was counted. The strips were divided into short endplatecontaining pieces and longer "extrajunctional" pieces, and their lengths were measured. The binding of ${ }^{125}-\alpha$-BuTx to NMJs and perijunctional areas was calculated by counting the radioactivity bound to lengths of muscle fibers containing endplates, subtracting background radioactivity associated with the same length of nonendplate portions of the same fibers, and dividing by the number of endplates. Extrajunctional AChR levels were determined by counting the radioactivity bound to lengths of nonendplate portions of muscle fibers and dividing by the length of the muscle fibers sampled. Specific binding was defined as the difference between the amounts of ${ }^{125} \mathrm{~F}-\alpha$-BuTx bound in the absence and in the presence of $40 \mu \mathrm{g} / \mathrm{ml}$ of unlabeled $\alpha$-BuTx. Internal AChR levels were determined in each muscle by subtracting the amount of specific binding of ${ }^{225} \mid-\alpha$-BuTx to nonpermeabilized muscle from the specific ${ }^{125} \mathrm{l}-\alpha$-BuTx binding to saponin-permeabilized pieces of the same muscle. Results are expressed as specific ${ }^{125} \mid-\alpha$-BuTx binding per NMJ or per unit length (micrometers) of muscle fiber.

Skeletal muscle cultures. Cultures of rat skeletal muscle were prepared by conventional methods (Yaffe, 1973), as previously described (Kao and Drachman, 1977; Drachman et al., 1982). Muscle cells $\left(5 \times 10^{4}\right)$ were plated per culture dish. They were incubated at $37^{\circ} \mathrm{C}$ under an atmosphere of $10 \%$ carbon dioxide in "standard medium" consisting of Eagle's minimum essential medium with Earle's salts, supplemented with $10 \%$ horse serum, 10 units/ $\mathrm{ml}$ of penicillin, 100 units $/ \mathrm{ml}$ of streptomycin, and $2.5 \mu \mathrm{g} / \mathrm{ml}$ of amphotericin $B$ (Fungizone). The number of AChR siles per culture was determined using ${ }^{125} \mid-\alpha$-BuTx binding. Eight to 10 days after plating, the cultures were fixed as above for $30 \mathrm{~min}$. Some were then treated with $0.5 \%$ saponin for $5 \mathrm{~min}$ and washed twice. AChRs were saturated with $0.2 \mu \mathrm{g} / \mathrm{ml}^{125} \mathrm{|}-\alpha$-BuTx (specific activity, 2 to $5 \times 10^{4} \mathrm{Ci} / \mathrm{mol}$ ) at $37^{\circ} \mathrm{C}$ for $30 \mathrm{~min}$, and the unbound toxin was removed by washing four times with wash medium. The cultures were then extracted with two 1 -mi washings with $2 \%$ Triton $X-100$ in phosphate-buffered saline, $\mathrm{pH} 7.2$, to solubilize the ${ }^{125} \mathrm{I}-\alpha$-BuTx-labeled AChRs, and the radioactivity in the extracts was counted. Results are reported as specific counts of ${ }^{125} \mathrm{I}-\alpha$-BuTx binding (defined as above) per cluture dish.

Immunological comparison of internal $\alpha$-BuTx binding sites with surface AChRs. Internal $\alpha$-BuTx binding sites and surface AChRs were extracted from cultured muscle fibers in order to compare their binding to anti-AChR antibody. Labeled surface AChRs were prepared by incubating unfixed muscle cultures with ${ }^{125} \mid-\alpha$-BuTX, washing, and extracting with Triton $X-100$, all as above. A preparation of labeled internal $\alpha$-BuTx-binding sites was obtained by (1) blocking surface AChRs with $1.0 \mu \mathrm{g} / \mathrm{ml}$ unlabeled $\alpha$-BuTx at $37^{\circ} \mathrm{C}$ for $30 \mathrm{~min}$; (2) washing and extracting as above; (3) labeling solubilized internal sites by adding a 3 to 10 -fold excess of ${ }^{125}$ - $\alpha$-BuTX; and (4) removing unbound ${ }^{125}-\alpha$-BuTx using a Sephadex G-200 column. Duplicate seria dilutions of rat serum containing high titers of anti-rat AChR anitbody were added to aliquots of surface AChRs and internal $\alpha$-BuTx binding sites, and the mixtures were incubated for $18 \mathrm{hr}$ at $4^{\circ} \mathrm{C}$. Antibody-receptor complexes were precipitated by addition of goat anti-rat IgG (Appel et al., 1975 Lindstrom et al., 1976). The percentage of radioactivity precipitated at each serum dilution was calculated. Specific antibody binding was then derived by subtracting the amount of nonspecific receptor precipitation in preparations with similar dilutions of control normal rat serum.

Statistical methods. Results are expressed as mean \pm standard error The significance of the differences between experimental groups was calculated by the Student's two-sample $t$ test.

\section{Results}

\section{$\alpha$-Bungarotoxin binding sites in normal young adult rat limb muscles}

Junctional sites. Innervated 4-month-old rat soleus muscle contained $1.6 \pm 0.1 \times 10^{7}$ AChRs/NMJ as measured by ${ }^{125}$ - $\alpha$-BuTx binding to fresh muscle. When measured immediately after fixation (see "Materials and Methods"), the number of AChRs/NMJ (1.7 \pm $0.2 \times 10^{7}$ ) was not significantly different (Table I). However, when membranes were permeabilized with saponin immediately before $\alpha$ BuTx binding, the number of apparent "junctional" AChRs approximately doubled to a total of $3.4 \pm 0.3 \times 10^{7}$ sites, an additional 1.7 $\pm 0.3 \times 10^{7} \alpha$-BuTx sites/NMJ. When fixed muscles were allowed to stand at $4^{\circ} \mathrm{C}$ for 1 week (another method of permeabilizing the membrane), the $\alpha$ BuTx binding increased to a similar degree (Table I). Permeabilization of the extensor digitorum longus (EDL) muscle
TABLE I

$\alpha$-BuTx binding sites in muscles from normal young adult rats Fresh and fixed muscles had similar numbers of AChRs/NMJ. Additional $\alpha$-BuTx binding sites were exposed by membrane permeabilization with saponin or muscle storage.

\begin{tabular}{llll}
\hline Muscle & \multicolumn{1}{c}{ Preparation } & $\begin{array}{c}\text { AChRs/NMJ } \\
\left(\times 10^{7}\right)\end{array}$ & $\begin{array}{c}\text { Internal } \alpha \text { BuTx } \\
\text { Binding Sites/ } \\
\text { NMJ } \\
\left(\times 10^{7}\right)\end{array}$ \\
\hline Soleus & Fresh (15) & $1.6 \pm 0.1^{\circ}$ & \\
& Fixed (20) & $1.7 \pm 0.2$ & \\
& Fixed and saponin & $3.4 \pm 0.3$ & $1.7 \pm 0.3$ \\
Soleus & Fixed (10) & $2.0 \pm 0.1$ & \\
& Fixed and stored & $3.5 \pm 0.4$ & $1.5 \pm 0.4$ \\
EDL & Fixed (5) & $1.8 \pm 0.1$ & \\
& Fixed and saponin & $3.3 \pm 0.4$ & $1.5 \pm 0.5$ \\
\hline
\end{tabular}

${ }^{a}$ Numbers in parentheses indicate number of muscles sampled.

${ }^{\circ}$ Results are expressed as mean \pm SEM.

membrane also revealed significant additional numbers of $\alpha$-BuTx binding sites (Table I). Autoradiography (Figs. 1 and 2) showed that the newly revealed sites were found inside the muscle fibers primarily in perijunctional regions within 200 to $300 \mu \mathrm{m}$ of the NMJ. There were few internal or surface sites beyond this region (Table II).

"Extrajunctional" sites. We compared the response of internal extrajunctional sites after denervation with the expected increase of surface extrajunctional AChRs (Table II). In control muscles, there were only $1.5 \pm 0.2 \times 10^{3} \mathrm{AChRs} / \mu \mathrm{m}$ of muscle fiber length on the surface membrane and $1.3 \pm 0.4 \times 10^{3}$ internal $\alpha$-BuTx sites $/ \mu \mathrm{m}$. After denervation surface and internal values rose to $16.2 \pm 1.5 \times$ $10^{3}$ and $16.9 \pm 2.8 \times 10^{3}$ sites/ $\mu \mathrm{m}$, respectively on day 4 , and to $25.8 \pm 1.9 \times 10^{3}$ and $22.5 \pm 2.0 \times 10^{3}$ sites $/ \mu \mathrm{m}$ on day 7 . Thus, surface and internal sites increased progressively and in parallel after denervation.

\section{Similarity of internal $\alpha$ BuTx-binding sites to AChRs}

Binding properties. We next compared the abilities of AChR blocking agents to bind to internal $\alpha$-BuTx sitcs and to AChRs on the muscle surface membrane. Initial experiments showed that internal sites, like AChRs, bind $\alpha$-BuTx in an irreversible manner. The amount of ${ }^{125} \mathrm{I}-\alpha$-BuTx bound to internal sites was similar whether muscles were washed for $5 \mathrm{hr}\left(1.7 \pm 0.2 \times 10^{7}\right.$ sites/NMJ) or $72 \mathrm{hr}$ $\left(1.6 \pm 0.1 \times 10^{7}\right.$ AChRs/NMJ). Preincubation with $40 \mu \mathrm{g} / \mathrm{ml}$ of unlabeled $\alpha$-BuTx blocked virtually all subsequent surface and internal binding of ${ }^{125}$-labeled $\alpha$-BuTx. Residual binding averaged $0.1 \pm$ $0.1 \times 10^{7}$ sites/NMJ for both internal and surface $\alpha$-BuTx binding sites (Table III).

Curare blocked internal and surface binding of $\alpha$-BuTx by similar proportions. Because $\alpha$-BuTx eventually displaces curare, this experiment was carried out at brief times of $\alpha$-BuTx binding ( $1 / 2 \mathrm{hr})$. Pretreatment of 7-day denervated soleus muscles for 15 min with 3 $\mu \mathrm{g} / \mathrm{ml}$ curare significantly inhibited $\alpha$-BuTx binding. Curare reduced extrajunctional surface binding of $\alpha$-BuTx by $76 \%(\rho<0.01)$ from $7.5 \pm 1.9 \times 10^{3}$ to $1.8 \pm 0.4 \times 10^{3}$ sites $/ \mu \mathrm{m}$. A similar degree of inhibition $(70 \%)(p<0.01)$ was seen on $\alpha$-BuTx binding to internal sites, reducing $\alpha$-BuTx binding from $12.6 \pm 4.8 \times 10^{3}$ to $3.8 \pm 1.3$ $\times 10^{3}$ sites $/ \mu \mathrm{m}$. These results show that AChR-blocking agents had similar effects on internal $\alpha$-BuTx binding sites and surface AChRs.

Metabolism. If internal $\alpha$-BuTx binding sites are precursors of surface AChRs en route to membrane insertion, then inhibition of protein synthesis should reduce inlerrial sites, earlier than it reduces surface AChRs (Patrick et al., 1977; Fambrough and Devreotes 1978). However, treatment with puromycin reduced surface AChRs and internal $\alpha$-BuTx binding sites to a similar degree (Fig. 3). Fifty microliters of $0.4 \mathrm{mg} / \mathrm{ml}$ puromycin were injected into 7-day denervated soleus muscles. After $4 \mathrm{hr}$ extrajunctional surface AChRs and internal sites were reduced to 95 and $93 \%\left(24.4 \pm 4.3 \times 10^{3}\right.$ and 

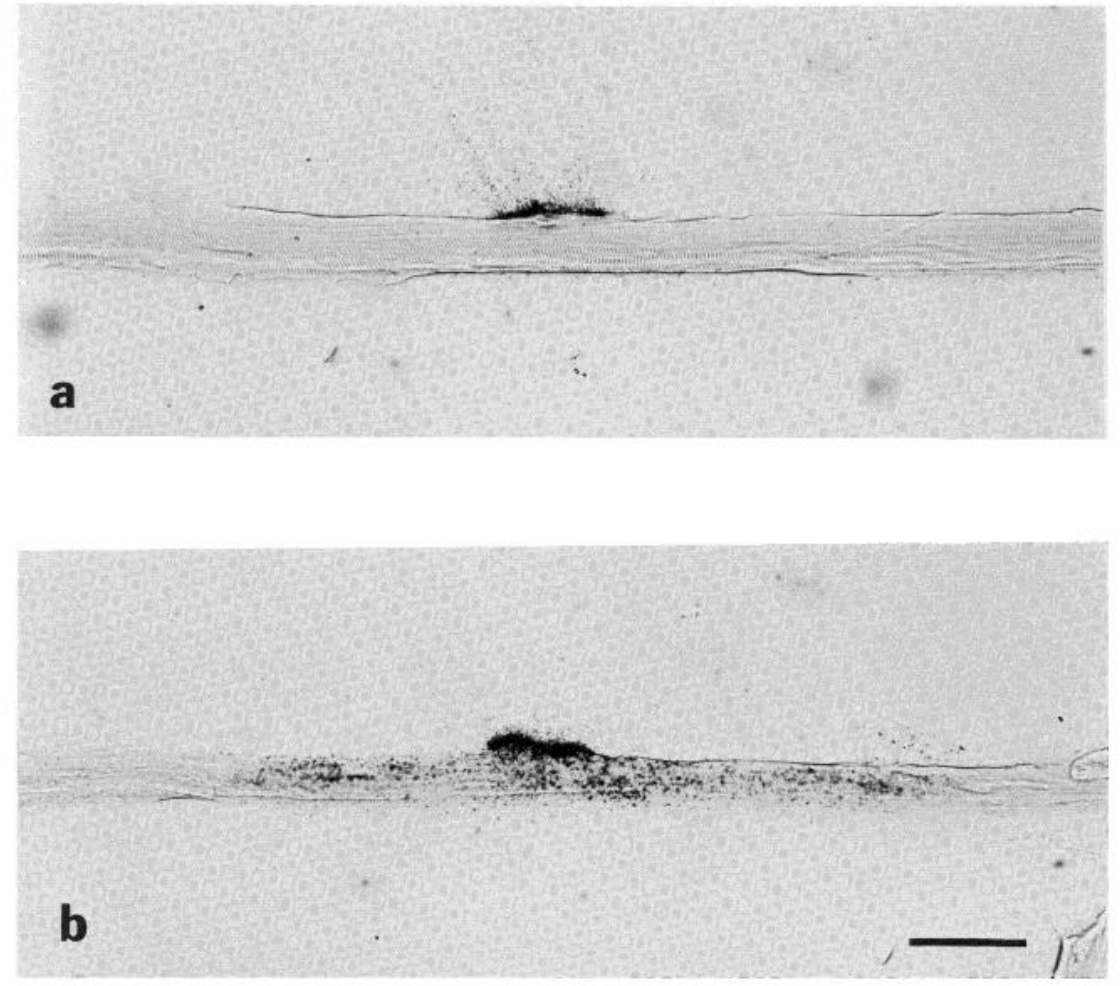

Figure 1. Autoradiography of ${ }^{125} \mathrm{~F}-\alpha$-BuTx binding to AChRs on teased muscle fibers in a normal soleus muscle from a 4-month-old rat. Bar $=80 \mu \mathrm{m}$. a, Surface AChRs at a neuromuscular junction. Silver grains are confined to the junctional area. $b$. Surface and internal AChRs from a normal soleus muscle. The membrane was permeabilized with saponin before exposure to ${ }^{125}{ }^{-\alpha}-$-BuTx. Note the appearance of perijunctional grains not seen when only surface AChRs are labeled.
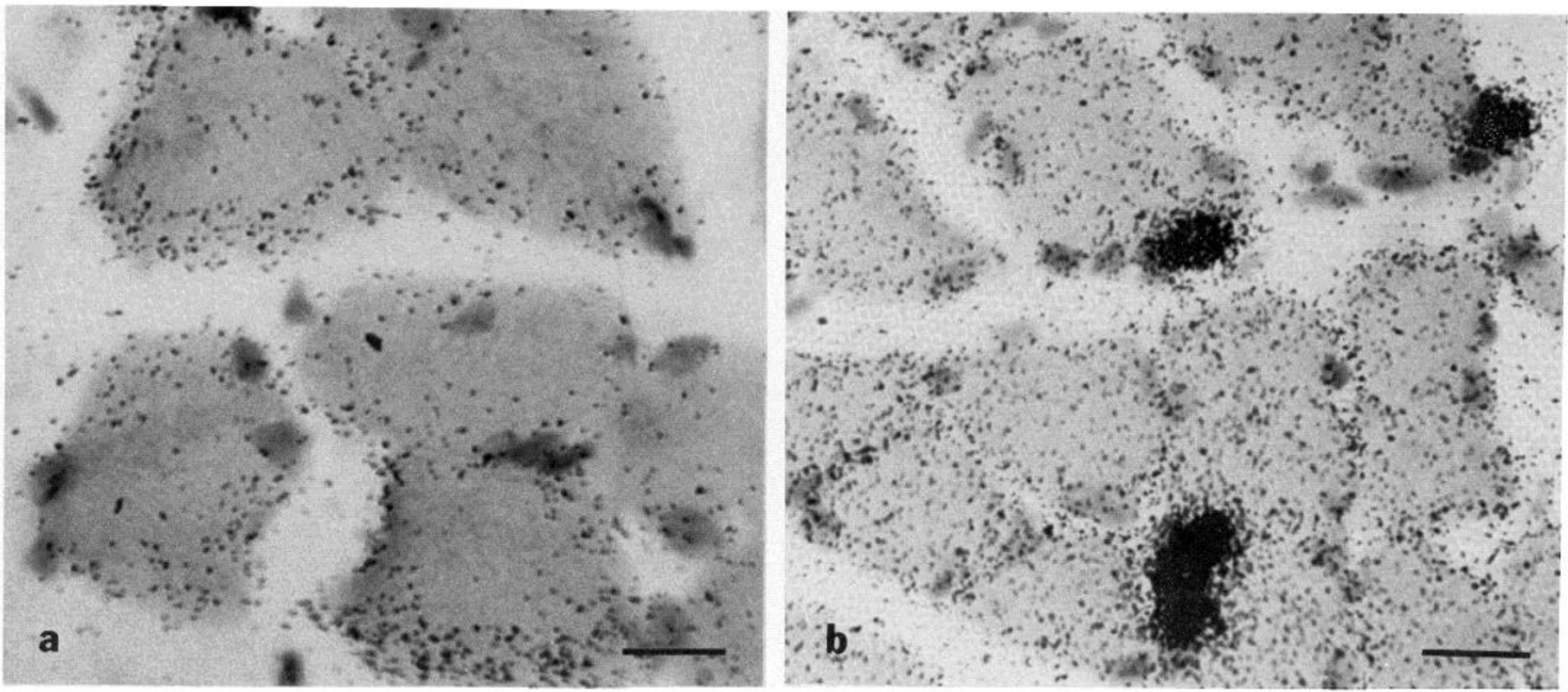

Figure 2. Autoradiography of ${ }^{125} \mathrm{~F}-\alpha$-BuTx binding to AChRs in a 7-day denervated soleus muscle. Muscles were tixed, exposed to ${ }^{125} \mathrm{~F}-\alpha$-BuTx, and cut in $10 \mu \mathrm{m}$-thick cross-sections. a, Surface AChRs on extrajunctional muscle membrane. Silver grains tend to form a ring along the surface of the fibers. Bar $=$ $20 \mu \mathrm{m}$. $b$, Surface and internal AChRs in muscles treated with saponin before exposure to ${ }^{125}-\alpha$-BuTx. Grains are located within the cell as well as on the surface of the fibers. Three neuromuscular junctions with dense accumulations of silver grains are located at the surface of muscle fibers. Bar $=30 \mu \mathrm{m}$.

$20.8 \pm 8.9 \times 10^{3}$ sites $\left./ \mu \mathrm{m}\right)$ of initial values, respectively; $22 \mathrm{hr}$ later, they were 74 and $78 \%\left(19.2 \pm 3.6 \times 10^{3}\right.$ and $17.5 \pm 3.8 \times 10^{3}$ sites $/ \mu \mathrm{m})$. This result does not rule out the idea that some internal sites are precursors of surface AChRs; however, it suggests that the majority are not (see culture experiments below).

\section{Independent variation of surface AChRs and internal $\alpha$-BuTx binding sites}

$\alpha$-BuTx blockade. Although the distribution and synthesis of surface AChRs and internal $\alpha$-BuTx binding sites have several similar characteristics, the two populations may vary independently in some circumstances. We first examined the short and longer-term effects of reducing surface $\alpha$-BuTx binding sites on the number of internal sites. Blockade of surface AChRs with $\alpha$-BuTx in vivo had no effect on internal $\alpha$-BuTx binding sites. In this experiment, control muscles had $1.7 \pm 0.3 \times 10^{7}$ surface AChRs/NMJ and $1.3 \pm 0.4 \times 10^{7}$ internal sites/NMJ (Table III). Three hours after injection of $0.2 \mathrm{mg} /$ $\mathrm{ml}$ of unlabeled $\alpha$-BuTx into the soleus muscle, the surface ${ }^{125} \mid-\alpha$ BuTx binding was reduced to $0.1 \pm 0.1 \times 10^{7} \mathrm{AChRs} / \mathrm{NMJ}$ but internal perijunctional sites remained unchanged at $1.2 \pm 0.3 \times 10^{7}$. 
TABLE ॥

Extrajunctional surface AChRs and internal $\alpha$-BuTx sites in the soleus muscle of 4-month-old rats

Note the parallel increase in both surface and internal sites after denervation.

\begin{tabular}{|c|c|c|}
\hline Experimental Treatment & $\begin{array}{l}\text { Surface AChRs } / \mu \mathrm{m} \\
\left(\times 10^{3}\right)\end{array}$ & $\begin{array}{c}\text { Internal } \alpha \text {-BuTx } \\
\text { Binding Sites/ } \\
\mu \mathrm{m} \\
\left(\times 10^{3}\right)\end{array}$ \\
\hline Control $(11)^{a}$ & $1.5 \pm 0.2^{b}$ & $1.3 \pm 0.2$ \\
\hline 4-day denervated (7) & $16.2 \pm 1.5$ & $16.9 \pm 2.8$ \\
\hline 7-day denervated (23) & $25.8 \pm 1.9$ & $22.5 \pm 2.0$ \\
\hline
\end{tabular}

${ }^{a}$ Numbers in parentheses indicate number of muscles sampled.

${ }^{0}$ Results are expressed as mean \pm SEM

\section{TABLE III}

Reduction of surface AChRs had no effect on internal $\alpha$-BuTx sites

Reduction of surface AChRs by $\alpha$-BuTx blockade or exposure to antiAChR antibody had no effect on internal $\alpha$-BuTx binding sites. Soleus muscles of 4-month-old rats were studied.

\begin{tabular}{lcc}
\multicolumn{1}{c}{ Experimental Procedure } & $\begin{array}{c}\text { Surface AChRs/NMJ } \\
\left(\times 10^{7}\right)\end{array}$ & $\begin{array}{c}\text { Internal } \alpha \text {-BuTx } \\
\text { Binding Sites/ } \\
\text { NMJ } \\
\left(\times 10^{7}\right)\end{array}$ \\
\hline $\begin{array}{l}\text { Control (8) } \\
\alpha \text {-BuTx block in vitro after sa- }\end{array}$ & $1.7 \pm 0.3^{\circ}$ & $1.3 \pm 0.4$ \\
$\quad$ ponin (5) & $0.1 \pm 0.1$ & $0.1 \pm 0.1$ \\
$\alpha$-BuTx in vivo-3 hr (5) & $0.1 \pm 0.1$ & $1.2 \pm 0.3$ \\
$\alpha$-BuTx in vivo-4 days (6) & $0.4 \pm 0.1$ & $1.2 \pm 0.2$ \\
Anti-AChR antibody (5) & $0.7 \pm 0.1$ & $1.2 \pm 0.2$ \\
\hline
\end{tabular}

a Numbers in parentheses indicate number of muscles sampled.

${ }^{\circ}$ Results are expressed as mean \pm SEM.

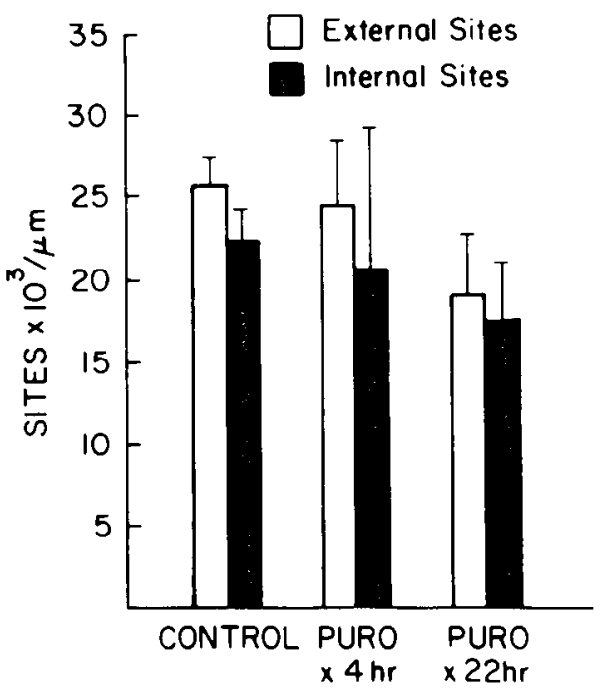

Figure 3. Effect of puromycin on surface and internal $\alpha$-BuTx binding sites in 7-day denervated soleus muscle. A single injection into the soleus muscle of $20 \mu \mathrm{g}$ of puromycin in $50 \mu \mathrm{l}$ of Ringer's solution produced a moderate decline in both internal and surface sites over a 22-hr period. Data are expressed as mean \pm SEM.

Four days after blockade surface junctional AChRs had partially recovered to $0.4 \pm 0.1 \times 10^{7}$, while internal sites were again unchanged at $1.2 \pm 0.2 \times 10^{7}$ (Table III). In muscles exposed to anti-AChR antibody, there is chronic damage to NMJs and an increased degradation rate of junctional AChRs, resulting in decreased numbers of surface AChRs. We examined soleus muscles from animals with high titers of anti-AChR antibody 5 weeks after immunization with AChR. Surface AChRs were reduced by $60 \%$ ( $\rho$ $<0.01$ ) to $0.7 \pm 0.1 \times 10^{7}$, while internal sites were unchanged at
$1.2 \pm 0.2 \times 10^{7}$. Thus, reducing surface $\alpha$-BuTx binding sites by blocking them or by increasing their internalization and degradation had no effect on internal sites.

Aging. There was a marked change in the relative distribution of surface AChRs and internal sites with aging (Table IV). In most animals up to 7 months of age, there were significant numbers of internal perijunctional binding sites. At 1 month, there were $0.3 \pm$ $0.1 \times 10^{7}$ internal sites per NMJ compared to $0.7 \pm 0.1 \times 10^{7}$ surface AChRs/NMJ. By 4 months of age, both values had increased to $1.7 \pm 0.3 \times 10^{7}$ and $1.7 \pm 0.2 \times 10^{7}$, respectively. Thereafter, surface AChRs/NMJ remained relatively constant, while internal sites declined (Table IV). A similar dissociation with aging was noted for extrajunctional surface AChRs and internal sites. In 4-month-old animals, surface AChRs and internal sites increased in parallel 7 days after denervation to $25.9 \pm 1.9 \times 10^{3}$ and $22.5 \pm 2.0 \times 10^{3}$ sites $/ \mu \mathrm{m}$, respectively. In 1 -year-old animals, surface AChRs increased to similar levels $\left(26.7 \pm 2.6 \times 10^{3}\right.$ sites $\left./ \mu \mathrm{m}\right) 7$ days atter denervation, but a significant increase of internal sites could not be detected $\left(1.1 \pm 1.2 \times 10^{3}\right.$ sites $\left./ \mu \mathrm{m}\right)$. Two experiments were carried out to control for the possibility that the reduction of internal $\alpha$-BuTx sites was due to reduced membrane permeability in older muscle fibers. Autoradiography of cross-sections of incompletely washed ${ }^{125}$ - $\alpha$-BuTx-treated specimens showed grains within the muscle fibers. In addition, longer exposure of muscles to a higher concentration of saponin ( $1 \%$ for 12 to $15 \mathrm{~min}$ ) failed to increase internal $\alpha$ BuTx binding. This suggests that the reduced internal $\alpha$-BuTx binding in older muscles is probably not an artifact secondary to poor permeabilization of membranes. Thus, in older animals, surface AChRs are unchanged, while internal $\alpha$-BuTx sites are markedly reduced even in denervated muscles actively synthesizing AChRs for insertion into the surface membrane. Histological examination of frocen cross-sections of older muscles revealed no obvious morphological differences from younger ones.

Internal sites in regenerating muscle. This experiment was designed to determine whether internal sites would reappear in older muscles that were damaged and allowed to regenerate. At the beginning of the experiment, surface AChRs and internal perijunctional sites averaged $2.1 \pm 0.2 \times 10^{7}$ and $-0.1 \pm 0.3 \times 10^{7}$ per NMJ, respectively, in soleus muscles from a group of 10-month-old rats (Table IV). The opposite soleus was then injected with $60 \mu \mathrm{l}$ of $3 \%$ Marcaine (bupivacaine $\mathrm{HCl}$, Sterling-Winthrop) solution (producing extensive muscle fiber necrosis). Five months later (a time several months after regeneration was complete, when muscle histology had returned virtually to normal), significant numbers of internal perijunctional sites had reappeared in the soleus, averaging $1.9 \pm$ $0.4 \times 10^{7}$ sites/NMJ. Surface AChRs/NMJ in these muscles measured $1.4 \pm 0.2 \times 10^{7}$. These results show that internal $\alpha$-BuTx sites reappear in the muscles of older animals after fiber damage and regeneration.

TABLE IV

\section{Aging and $\alpha$-BuTx binding sites}

The number of surface AChRs remained unchanged from 4 to 18 months of age. Internal sites were abundant in younger animals but disappeared with increasing age. These sites reappeared if muscles were damaged and allowed to regenerate.

\begin{tabular}{ccc}
\hline $\begin{array}{c}\text { Age } \\
\text { (months) }\end{array}$ & $\begin{array}{c}\text { Surface AChRs/NMJ } \\
\left(\times 10^{7}\right)\end{array}$ & $\begin{array}{c}\text { Internal } \alpha \text {-BuTx } \\
\text { Binding Sites/ } \\
\text { NMJ } \\
\left(\times 10^{7}\right)\end{array}$ \\
\hline 1 & $0.7 \pm 0.1^{a}(8)^{b}$ & $0.3 \pm 0.1$ \\
4 & $1.7 \pm 0.2(20)$ & $1.7 \pm 0.3$ \\
7 & $1.8 \pm 0.2(6)$ & $1.2 \pm 0.7$ \\
10 & $2.1 \pm 0.2(6)$ & $-0.1 \pm 0.3$ \\
13 & $1.6 \pm 0.4(6)$ & $0.0 \pm 0.5$ \\
18 & $2.0 \pm 0.3(14)$ & $0.0 \pm 0.2$ \\
\hline
\end{tabular}

\footnotetext{
${ }^{a}$ Results are expressed as mean \pm SEM.
}

${ }^{b}$ Numbers in parentheses indicate number of muscles sampled. 


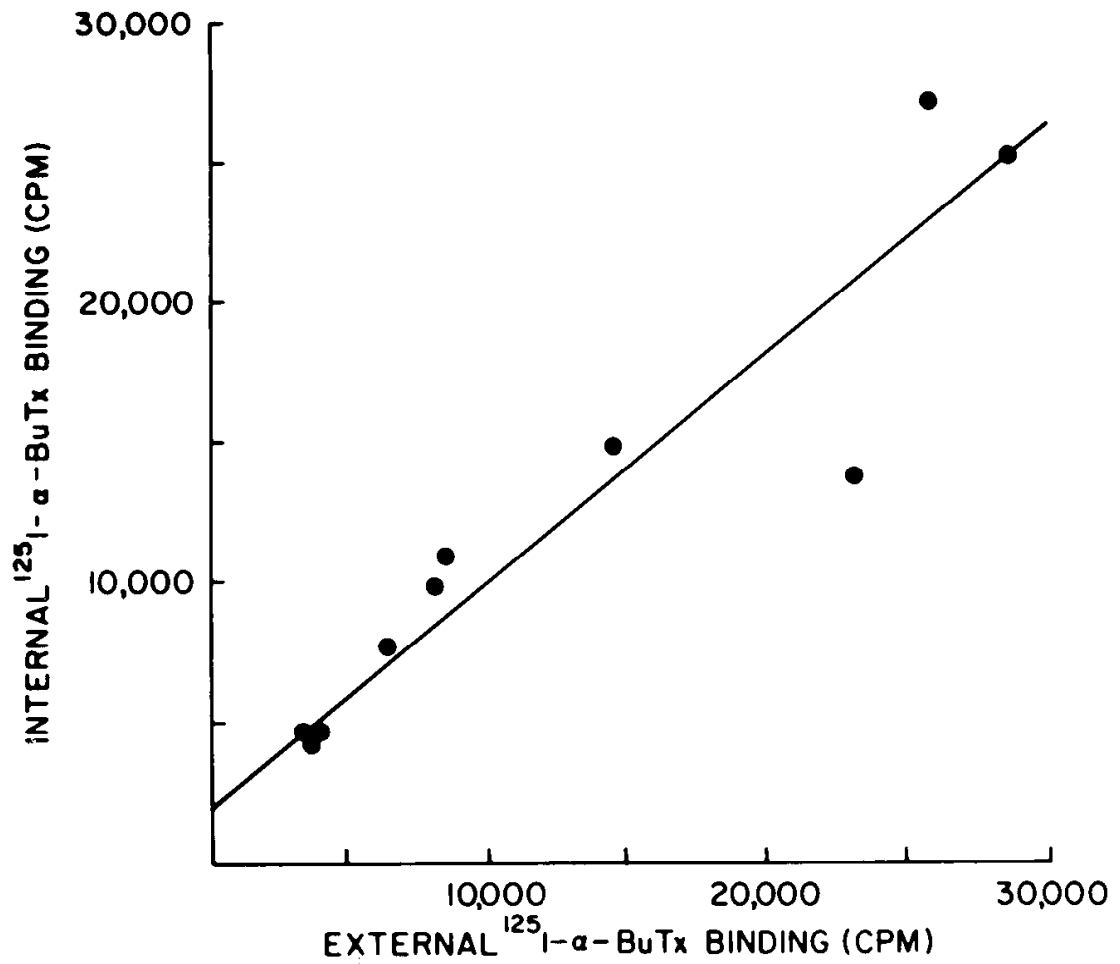

Figure 4. Relationship between surface and internal $\alpha$ BuTx binding in skeletal muscle in vitro. Cultured muscle, like muscle from young adult animals in vivo, had similar numbers of surface and internal AChRs. Each data point represents the mean of specific ${ }^{125}-\alpha$-BuTx binding to 10 culture dishes.

Internal sites in cultured rat skeletal muscle

Our studies of young rat muscle in vivo showed almost equal numbers of surface AChRs and internal $\alpha$-BuTx binding sites. In contrast, previous in vitro studies suggested that internal sites were relatively scarce (Fambrough and Devreotes, 1978)-33\% of surface AChR levels. We therefore examined internal $\alpha$-BuTx binding sites in cultured rat skeletal muscle. Our results showed that, as for our in vivo results, internal sites and surface AChRs were present in similar numbers. The difference in the number of internal sites between this and previous studies could be explained by the fact that muscle fibers in the present experiments were not exposed to $\alpha$-BuTx until the time of assay. In previous studies, muscle fibers were grown in the presence of unlabeled $\alpha$-BuTx. In our experiments, the apparent number of internal sites was reduced by $49 \%$ in muscle fibers cultured for 3 to 5 days with unlabeled $\alpha$-BuTx before assay with ${ }^{125} \mid-\alpha$-BuTx.

As with $\alpha$-BuTx binding in vivo, surface and internal sites in vitro varied in parallel; cultures with high levels of surface AChRs had high internal levels was well (Fig. 4). Levels of both surface AChRs and internal sites were both increased after addition of tetrodotoxin

TABLE V

Effects of pharmacological treatments on surface and internal AChRs in vitro

Forty-eight-hour treatment of cultures with tetrodotoxin, an agent that blocks sodium channels and eliminates muscle fiber activity, increased both internal and surface AChRs. Exposure of muscle to puromycin for $5 \mathrm{hr}$ to block AChR synthesis significantly reduced internal AChRs but produced only a small change in surface AChRs.

\begin{tabular}{lll}
\hline \multicolumn{1}{c}{ Treatment } & \multicolumn{1}{c}{ Surface Sites } & \multicolumn{1}{c}{ Internal Sites } \\
\hline Control $(20)^{a}$ & $6280 \pm 97^{b}$ & $7765 \pm 404$ \\
$\begin{array}{l}\text { Tetrodotoxin (10) (in- } \\
\quad \text { crease) }\end{array}$ & $7264 \pm 201(16 \%)$ & $9727 \pm 685(25 \%)$ \\
$\begin{array}{l}\text { Puromycin (10) (in- } \\
\text { crease) }\end{array}$ & $5746 \pm 123(-9 \%)$ & $6010 \pm 231(-23 \%)$ \\
\end{tabular}

\footnotetext{
${ }^{a}$ Numbers in parentheses indicate number of cultures sampled.

${ }^{b}$ Results are $\mathrm{cpm}$ of ${ }^{125} \mathrm{~F}-\alpha$-BuTx binding per culture dish, expressed as mean \pm SEM
}

$\left(10^{-6} \mathrm{M}\right)$ to cultures for $48 \mathrm{hr}($ Table V). However, puromycin $(20 \mu \mathrm{g} /$ $\mathrm{ml}$ ) treatment of cultures for $5 \mathrm{hr}$ significantly reduced internal sites by $23 \%$, while surface AChRs fell only $9 \%$ (Table V). This suggests that approximately 14 to $23 \%$ of the internal sites in muscle in vitro are similar to the "newly synthesized" AChRs previously demonstrated in other studies.

\section{Immunological comparison of internal $\alpha$-BuTx binding sites with surface AChRs}

We used an immunoprecipitation assay to compare the binding of anti-AChR antibody to surface AChRs and internal $\alpha$-BuTx binding sites (Fig. 5). At a low relative concentration of anti-AChR antibody, there was a 3-fold difference in the precipitation of internal sites $(23 \%)$ and surface AChRs (72\%). However, this difference narrowed with increasing amounts of antibody. At the highest concentration of antibody, a similar degree of internal sites (82\%) and surface AChRs $(92 \%)$ was precipitated. Thus, although there seemed to be antigenic differences between the two preparations, both surface AChRs and internal $\alpha$-BuTx binding sites were recognized by antiAChR antibody.

\section{Discussion}

Characterization of internal $\alpha$-BuTx binding sites. In this study, we have examined $\alpha$-BuTx binding sites within adult rat skeletal muscles in vivo and compared the results with those from cultured rat skeletal muscle. Internal sites were exposed by permeabilizing the fixed cell membrane with a detergent and were labeled using ${ }^{125} \mid-\alpha$-BuTx. Our data show that internal sites are present in large numbers, both in developing and young adult rat muscle. These internal sites have several properties in common with AChRs on the surface membrane. They are probably analogous to the internal AChRs previously identified in cultured (Patrick et al., 1977; Fambrough and Devreotes, 1978) and developing muscle (Atsumi, 1981), although we have found that they occur in significantly larger numbers. The sites are not an artifact of exposure to saponin, since permeabilizing the membrane by 1 to 7 -day storage of the fixed muscle, without detergent, reveals similar numbers of internal sites.

The internal $\alpha$-BuTx binding siles in skeletal muscle are probably AChRs. (The ability of a cell to bind $\alpha$-BuTx does not prove it 
rigure 5. Comparison of immunoprecipitation of surface AChRs and internal $\alpha$-BuTx binding sites. At high concentrations, anti-AChR antibody recogrized both molecules which suggests that both are AChRs. However, at lower concentrations of antibody more surface than internal receptors were precipitated, which suggests that there are antigenic differences between them. In each assay, $50 \mu$ l of diluted serum were added to $250 \mu$ lof the ${ }^{125} /$ - $\alpha$-BuTx-receptor preparation. Serum dilutions ranged from 1:200 to undiluted. Results were expressed as the percentage of ${ }^{125} \mathrm{l}-\alpha$ BuTx-labeled receptor precipitated. At each serum concentration, surface AChR and internal sile data were calculated by subtracting nonspecific counts precipitated in the presence of normal rat serum from counts precipitated by anti-AChR antibody. Each data point represents the mean of two assays.

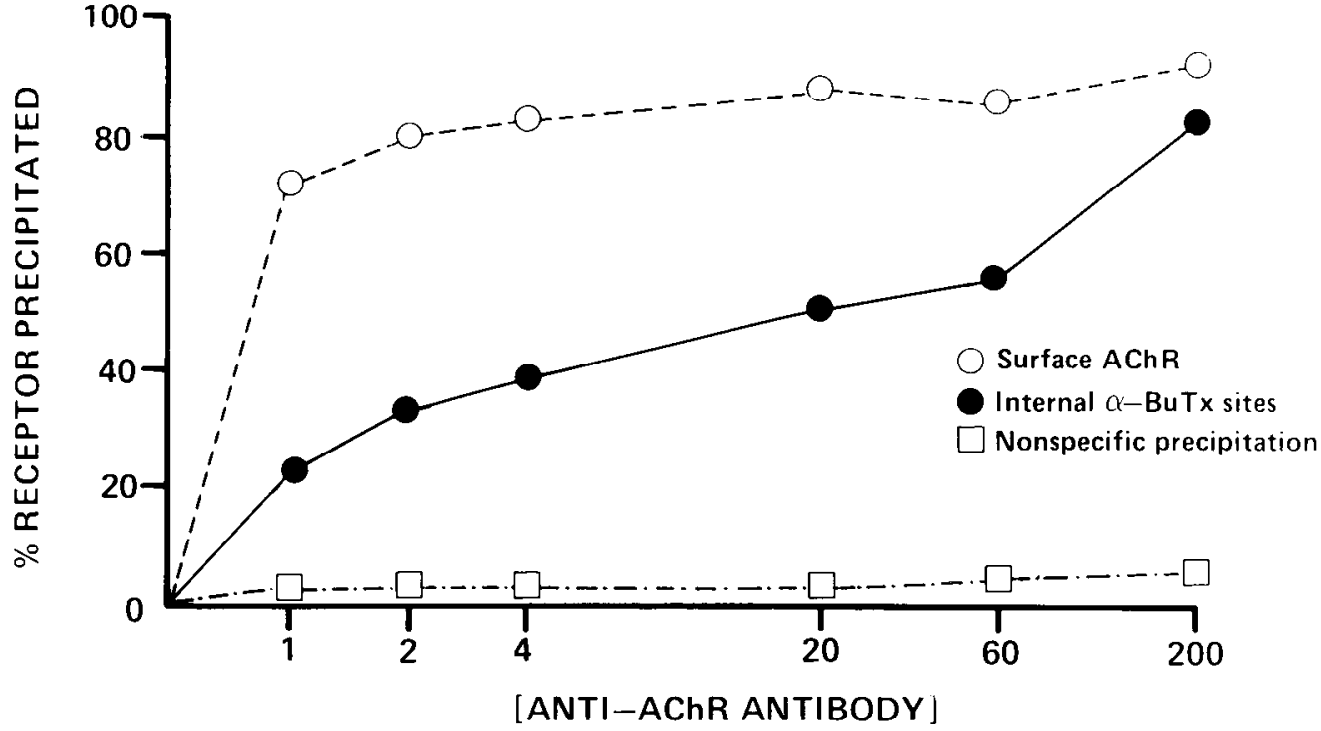

contains AChRs. For example, neuronal $\alpha$-BuTx binding sites may be related to but not identical with AChRs (Nurse and O'Lague, 1975; Brown and Fumagalli, 1977; Patrick and Stallcup, 1977; Carbonetto et al., 1978; Ravdin et al., 1981; Jacob and Berg, 1983)). The results of our study support the idea that internal sites are AChRs. In young muscle, surface AChRs and internal sites have a similar distribution. Both are present at or near the NMJ in normal muscle and appear in extrajunctional regions after denervation. The binding of $\alpha$-BuTx to internal sites is specific and saturable. Preexposure of internal sites to unlabeled $\alpha$-BuTx completely inhibits subsequent binding of ${ }^{125} /-\alpha$-BuTx. Anti-AChR antibody recognizes the internal $\alpha$-BuTx binding siles. Further evidence that internal sites are AChRs is provided by the curare blocking data. Preexposure of internal sites and surface AChRs to curare blocks binding of $\alpha$-BuTx to both, to the same degree. Finally, other studies have shown by a variety of techniques that intracellular $\alpha$-BuTx binding sites in skeletal muscle seem similar to cell surface AChRs (Devreotes et al., 1977; Patrick et al., 1977). $\alpha$-BuTx binding sites that are detergent-extracted from skeletal muscle show single peaks for junctional and extrajunctional sites on zone sedimentation and isoelectric focusing (Brockes and Hall, 1975)

The internal AChRs are not simply newly exposed sites on existing surface AChRs. The antibody binding studies suggest that there are antigenic differences between the two. In addition, autoradiography of muscles shows that surface and internal AChRs have a different distribution. In innervated muscle, surface AChRs are located at the NMJ, in a small area 20 to $50 \mu \mathrm{m}$ in length, whilo internal AChRs are also found in perijunctional regions within 200 to $300 \mu \mathrm{m}$ of the NMJ. Cross-sections of muscle show that the internal AChRs are distributed within muscle fibers, while exposed AChRs are located only at the surface.

Rolationship betwcen intornal and surface AChRs. Previous studies have found that the number of internal AChRs in skeletal muscle was about 20 to $33 \%$ that of surface AChRs (Fambrough and Devreotes, 1978). They suggested that many of these AChRs were newly synthesized and being transported, over a period of $3 \mathrm{hr}$, to the cell surface, where they are endocytosed and degraded, with a half-life of about 20 to $30 \mathrm{hr}$. It has also been shown that some internal AChRs are former surface AChRs, endocytosed and apparently en route to lysosomal degradation (Fumagalli et al., 1982). There is no evidence for internal recycling of endocytosed AChRs back into the surface membrane.

It appears from our data that the majority of the internal AChRs in rat skeletal muscle are not newly synthesized precursors of surface AChRs. If most internal AChRs were presursors of surface molecules, then one would predict that a reduction in internal AChRs would be followed by a similar change on the surface. In fact, the loss of internal AChRs with aging occurs without a significant reduction of surface AChRs. In addition, there may be as many internal AChRs as surface ones, far more than the 10 to $15 \%$ of surface AChRs that could be explained simply by a 3-hr supply of newly synthesized AChRs. In vitro studies show that inhibition of protein synthesis reduces "newly synthesized" internal AChRs by 80 to $90 \%$ within $3 \mathrm{hr}$ (Devreotes and Fambrough, 1975; Patrick et al., 1977). In contrast, our results show that a 5 -hr inhibition of protein synthesis has only a small effect on the total number of internal AChRs. Our culture results suggest that newly synthesized AChRs comprise about 14 to $23 \%$ of the internal pool. This figure may be considerably lower at the neuromuscular junction in vivo, where AChRs may have a significantly longer average half-life.

It also seems unlikely that most of the internal AChRs result from endocytosis of surface AChRs. Blockade of surface AChRs with $\alpha$ BuTx does not reduce internal AChRs after either several hours or days are allowed for equilibration. Similarly, there is no change in internal AChRs when surface AChRs are reduced by an antibodyinduced acceleration of their endocytosis.

Conclusions. It seems likely that the internal $\alpha$-BuTx binding sites demonstrated in this study are AChRs. This large population of internal molecules (AChRs) usually associated with the surface membrane seems similar to the internal sodium channels found in frog myocardial cytosol (Doyle et al., 1982). The function of such internal receptors remains unclear; however, they could represent some form of oversynthesis of AChRs that becomes more precisely regulated in later life. If so; it suggests that the muscle cell possesses a postsynthetic mechanism that controls the number of AChRs inserted into the cell membrane. Eventually, however, AChR synthesis itself seems to become more precisely regulated, as reflected by the disappearance of most internal AChRs after 6 to 8 months. This change provides an interesting intracellular analogy to the loss of "supernumerary" cells or cell processes during the development of the nervous system (Cowen et al., 1984).

Our results show that motor nerves can induce localization not only of surface AChRs to the NMJ but also of internal AChRs to perijunctional regions. This could reflect localized perijunctional synthesis of AChRs within innervated muscle fibers or intracellular transport to the NMJ of AChRs synthesized all along the muscle cell. In either case, it will be interesting to define the mechanisms by which the small surface neuromuscular contact controls the organization of molecules within a considerably larger intracellular space.

The place of internal AChRs in the overall metabolic pathway of AChRs is still undefined. It is possible that they are not inserted into the surface membrane because of some difference in their structure. 
Biochemical characterization of these AChRs is in progress. In any case, most internal AChRs do not appear to be essential to cellular function, since their number is greatly reduced in older muscle. This reduction with time provides a marker for cellular age. Younger or newly regenerated cells have numerous internal AChRs, while older muscle fibers have few. It will be interesting to determine whether the disappearance of AChRs with age has a morphological counterpart, since AChRs are usually thought to be associated with membranous organelles such as the Golgi apparatus and the surface membrane. (Our preliminary studies confirm that most of the internal AChRs seem to be associated with membrane). Wherever they are located, our results show that a significant proportion of transmitterreceptors in cells may not be functional surface molecules. Future studies, particularly those showing receptor changes with aging should take this factor into account.

\section{References}

Albuquerque, E. X., E. A. Barnard, C. W. Porter, and J. E. Warnick (1974) The density of acetylcholine receptors and their sensitivity in the postsyn aptic membrane of muscle endplates. Proc. Natl. Acad. Sci. U. S. A. 71 : 2818-2822.

Appel, S. H., R. R. Almon, and N. Levy (1975) Acetylcholine receptor antibodies in myasthenia gravis. N. Engl. J. Med. 293: 760-761.

Atsumi, S. (1981) Localization of surface and internal acetylcholine receptors in developing fast and slow muscles of the chick embryo. Dev. Biol. 86: $122-135$.

Barnard, E. A., J. Wieckowski, and T. H. Chiu (1971) Cholinergic receptor molecules and cholinesterase molecules at mouse skeletal muscle junctions. Nature 234: 207-209.

Brockes, J. P., and Z. W. Hall (1975) Acetylcholine receptors in normal and denervated rat diaphragm muscle. II. Comparison of junctional and extrajunctional receptors. Biochemistry 14: 2100-2106.

Brown, D. A., and L. Fumagalli (1977) Dissociation of $\alpha$ bungarotoxin binding and receptor block in the rat superior cervical ganglion. Brain Res. 129: $165-168$.

Carbonetto, S. T., D. M. Fambrough, and K. J. Muller (1978) Nonequivalence of $\alpha$-bungarotoxin receptors and acetylcholine receptors in chick sympathetic neurons. Proc. Natl. Acad. Sci. U. S. A. 75: 1016-1020.

Chang, C. C. , S. T. Chuang, and M. C. Huang (1975) Effects of chronic treatment with with various neuromuscular blocking agents on the number and distribution of acetylcholine receptors in the rat diaphragm. J. Physio (Lond.) 250: 161-173.

Cowan, W. M., J. W. Fawcett, D. D. M. O'Leary, and B. B. Stanfield (1984) Regressive events in neurogenesis. Science 22.5: 1258-1265.

Daniels, M. P., and Z. Vogel (1975) Immunoperoxidase staining of $\alpha$ bungarotoxin binding sites in muscle endplates shows distribution of acetylcholine receptor. Naturc 254: 339341

Devreotes, P. N., and D. M. Fambrough (1975) Acetylcholine receptor turnover in membranes of developing muscle fibers. J. Cell Biol. 65: 335358.

Devreotes, P. M., J. M. Gardner, and D. M. Fambrough (1977) Kinetics of biosynthesis of acetylcholine receptor and subsequent incorporation into plasma membrane of cultured chick skeletal muscle. Cell 10: 365-373.
Doyle, D. D., M. Wong, J. Tanaka, and L. Barr (1982) Saxitoxin binding sites in frog myocardial cytosol. Science 211: 1117-1119.

Drachman, D. B., R. N. Adams, L. F. Josifek, S. G. Self (1982) Functional activities of anti-AChR autoantibodies and clinical severity of myasthenia gravis. N. Engl. J. Med. 307: 769-775.

Fambrough, D. M., and P. N. Devreotes (1978) Newly synthesized acetylcholine receptors are located in the Golgi apparatus. J. Cell Biol. 76: $237-$ 244.

Fambrough, D. M., and H. C. Hartzell (1972) Acetylcholine receptors: number and distribution at neuromuscular junctions in rat diaphragm. Science 176. 189-191.

Fertuck, H. C., and M. M. Salpeter (1974) Localization of acetylcholine receptor by ${ }^{125}$-labeled $\alpha$-bungarotoxin binding at mouse motor endplates. Proc. Natl. Acad. Aci. U. S. A. 71: 1376-1378.

Fumagalli, G., A. G. Engel, and J. Lindstrom (1982) Ultrastructural aspects of acetylcholine receptor turnover at the normal end-plate and in autoimmune myasthenia gravis. J. Neuropathol. Exp. Neurol. 41: 567-579.

Hartzell, H. C., and D. M. Fambrough (1972) Acetylcholine receptors. Distribution and extrajunctional density in rat diaphragm after denervation correlated with acetylcholine sensitivity. J. Gen. Physiol. 60: 248-262.

Jacob, M. H. and D. K. Berg (1983) The ultrastructural localization of $\alpha$ bungarotoxin binding sites in relation to synapses on chick ciliary ganglion neurons. J. Neurosci. 3: 260-271.

Kao, I., and D. B. Drachman (1977) Myasthenic immunoglobulin accelerates acetylcholine receptor degradation. Science 195: 74-75.

Lee, C.Y. (1972) Chemistry and pharmacology of polypeptide toxins in snake venoms. Annu. Rev. Pharmacol. 12: 265-286.

Libelius, R. (1974) Binding of ${ }^{3} \mathrm{H}$-labeled cobra neurotoxin to cholinergic receptors in fast and slow mammalian muscles. J. Neural Transm. 35: $137-149$

Lindstrom, J. M., M. E. Seybold, V. A. Lennon, and S. Whittingham (1976) Antibody to acetylcholine receptor in myasthenia gravis: Prevalence, clinical correlates, and diagnostic value. Neurology (Minneap.) 26: 1054-1059.

Miledi, R. and L. T. Potter (1971) The acetylcholine receptors in muscle fibers. Nature 233: 599-603.

Nurse, C. A. and P. H. O'Lague (1975) Formation of cholinergic synapses between dissociated sympathetic neurons and skeletal myotubes of the rat in cell culture. Proc. Nati. Acad. Sci. U. S. A. 72: 1955-1959.

Patrick, J., and W. B. Stallcup (1977) Immunological distinction between acetylcholine receptor and the $\alpha$-bungarotoxin binding component on sympathetic neurons. Proc. Natl. Acad. Sci. U. S. A. 74: 4689-4692

Patrick, J., J. McMillan, H. Wolfson, and J. C. O'Brien (1977) Acetylcholine receptor metabolism in a nonfusing muscle cell line. J. Biol. Chem. 252 2143-2153.

Pestronk, A., D. B. Drachman, and J. W. Griffin (1976) Effect of muscle disuse on acetylcholine receptors. Nature 260: 352-353.

Pestronk, A., D. B. Drachman, R. Teoh, and R. N. Adams (1983) Combined short-term immunotherapy for experimental autoimmune myasthenia gravis. Ann. Neurol. 14: 235-341.

Ravdin, P. M., R.M. Nitkin, and D. K. Berg (1981) Internalization of $\alpha$ bungarotoxin on neurons induced by a neurotoxin that blocks neuronal acetylcholine sensitivity. J. Neurosci. 1: 849-861.

Yaffe, D. (1973) Rat skeletal muscle cells. In Tissue Culture: Methods and Applications, P. Kruse, Jr., and M. K. Paterson, eds., pp. 106-113, Academic Press, Inc., New York. 